\title{
Posterior Lumbar Plexus Block in
} Postoperative Analgesia for Total Hip Arthroplasty. A Comparative Study between $0.5 \%$ Bupivacaine with Epinephrine and 0.5\% Ropivacaine

(Rev Bras Anestesiol, 2009;59:273-285)

With considerable interest we read the article "Posterior Lumbar Plexus Block in Postoperative Analgesia for Total 
Hip Arthroplasty. A Comparative Study between 0.5\% Bupivacaine with Epinephrine and 0.5\% Ropivacaine." published in this journal by Duarte et al. ${ }^{1}$. The authors concluded that $0.5 \%$ ropivacaine and $0.5 \%$ bupivacaine produced effective and prolonged pain relief after total hip arthroplasty, without clinical differences, when equivalent doses were administered. However, the measured VAS scores were significantly lower in the Ropivacaine group on 8,12 and 24 hours postpuncture compared with the Bupivacaine group. Results from a similar study performed by our group ${ }^{2}$, however, differ in some ways,compared to the results of Duarte et al. Although our VAS scores were slightly higher, only $11 \%$ of the studied patients needed rescue opioids, probably suggesting an analgesic potency factor of the sciatic nerve block for this kind of surgery. Most remarkable differences were found in the VAS scores of the Ropivacaine group. In Duarte's study, $136 \mathrm{mg}$ Ropivacaine (mean) was injected in the psoas compartment compared to $180 \mathrm{mg}$ Ropivacaine (combined with $45 \mathrm{mg}$ in the proximity of the sciatic nerve) in our study. However, the VAS scores within the Ropivacaine group in Duarte's study (median $=0$ ) were significantly lower than the VAS scores within the Ropivacaine group of our study. In our opinion, this difference cannot not be explained by a cumulative consumption of $10.2 \mathrm{mg}$ of morphine the first 24 hours in Duarte's study or a difference in the approach of the posterior lumbar plexus block (Capdevila's vs Chayen).

There remains considerable debate about equipotency of Ropivacaine and Bupivacaine for peripheral nerve blocks. In our opinion, as local anesthetics are in general over-dosed, the term "equipotent dose" in peripheral nerve blocks becomes less accurate. Further studies concerning local anesthetic dose reductions for (ultrasound guided) peripheral nerve blocks are needed to provide us more reliable information about equipotency of long acting local anesthetics used in peripheral nerve blocks.

Marcel A. de Leeuw

Department of Anesthesiology, Intensive Care and Pain Medicine. Zaans Medical Centre. Zaandam. The Netherlands

Roberto GSM Perez

Wouter WA Zuurmond

Department of Anesthesiology. VU University Medical Centre. Amsterdam. The Netherlands

\section{RÉPLICA}

I would like to thank Leeuw et al. for their interest in our study. I also understand that the discussion regarding equipotent doses of bupivacaine and ropivacaine in peripheral nerve blocks is still open and requires further studies. The main ob- jective of our study was to compare the clinical impact of the administration of similar doses and concentration of those local anesthetics in psoas compartment block. Therefore, we tested the hypothesis that bupivacaine and ropivacaine would be equipotent and produce comparable analgesia after total hip arthroplasty.

One should be very careful when comparing the results of our study to those of Leeuw et al. Although the conclusion of both studies was similar, i.e., that bupivacaine and ropivacaine would produce effective and prolonged pain relief after total hip arthroplasty, in the study of Leeuw et al. different concentrations and doses of the local anesthetics were administered and they were also greater than the ones used in our study. Besides, the combination of sciatic nerve block most likely makes it impossible to compare both studies. Although lumbar plexus block is the main factor responsible for postoperative pain control, the blockade of the sacral component optimizes analgesia, especially in the first hours after hip arthroplasty, and it can explain the lower consumption of morphine in the stud of Leeuw et al. Finally, the addition of adrenaline $\left(5 \mu \mathrm{g} \cdot \mathrm{mL}^{-1}\right)$ to bupivacaine can also be a source of criticism in the study on equipotent doses of both drugs, since it can add to the analgesic effect. Differences in the design of both studies can explain the differences observed in postoperative pain scores and and opioid consumption.

In our opinion, we cannot conclude, definitely, that those two anesthetics are equipotent in psoas compartment blocks based only on the results of clinical studies like those mentioned here. A well-fundamented conclusion can only be achieved after a study that determines and compares the minimal anesthetic doses of those drugs in psoas compartment blocks. Still, different parameters con be evaluated, such as the extension of the blockade, the degree of the blockade, or postoperative pain relief. However, I am not aware of any study of this type on psoas compartment block.

Leonardo T. D. Duarte Rede SARAH of Rehabilitation Hospitals

\section{REFERÊNCIAS - REFERENCES}

01. Duarte LTD, Paes FC, Fernandes MCBC et al. - Posterior lumbar plexus block in postoperative analgesia for total hip arthroplasty. A comparative study between $0.5 \%$ Bupivacaine with epinephrine and 0.5\% ropivacaine. Rev Bras Anestesiol 2009;59:273-285.

02. Leeuw de MA, Dertinger D, Hulshoff L et al. - The efficacy of levobupibacaine, ropivacaine, and bupivacaine for combined psoas compartment - sciatic nerve block in patients undergoing total hip arthroplasty. Pain Practice 2008;8:214-247. 